\title{
UNIFORM BOUNDS FOR PREPERIODIC POINTS IN FAMILIES OF TWISTS
}

\author{
ALON LEVY, MICHELLE MANES, AND BIANCA THOMPSON \\ (Communicated by Matthew A. Papanikolas)
}

\begin{abstract}
Let $\phi$ be a morphism of $\mathbb{P}^{N}$ defined over a number field $K$. We prove that there is a bound $B$ depending only on $\phi$ such that every twist of $\phi$ has no more than $B K$-rational preperiodic points. (This result is analogous to a result of Silverman for abelian varieties.) For two specific families of quadratic rational maps over $\mathbb{Q}$, we find the bound $B$ explicitly.
\end{abstract}

\section{INTRODUCTION}

Let $K$ be a number field and $\phi: \mathbb{P}^{N}(K) \rightarrow \mathbb{P}^{N}(K)$ be a morphism on projective space of dimension $N$. (Note: When the ground field is clear we write simply $\mathbb{P}^{N}$.) We denote by $\phi^{n}$ the $n^{\text {th }}$ iterate of $\phi$ under composition. A point $P \in \mathbb{P}^{N}$ is periodic (with period $n$ ) if there exists an integer $n>0$ such that $\phi^{n}(P)=P$, and $P$ has primitive period $n$ if $n$ is the smallest such number. Also, $P$ is preperiodic if there exist integers $n>m \geq 0$ such that $\phi^{n}(P)=\phi^{m}(P)$. Let

$$
\operatorname{PrePer}\left(\phi, \mathbb{P}^{N}(K)\right)=\left\{P \in \mathbb{P}^{N}(K): P \text { is preperiodic under } \phi\right\} .
$$

A motivating problem in the field of arithmetic dynamics is the uniform boundedness conjecture of Morton and Silverman [10].

Conjecture 1. Let $K / \mathbb{Q}$ be a number field of degree $D$, and let $\phi: \mathbb{P}^{N} \rightarrow \mathbb{P}^{N}$ be a morphism of degree $d \geq 2$ defined over $K$. There is a constant $\kappa(D, N, d)$ such that

$$
\# \operatorname{PrePer}\left(\phi, \mathbb{P}^{N}(K)\right) \leq \kappa(D, N, d) .
$$

This is a deep and difficult problem. It implies, for example, uniform boundedness for torsion points on abelian varieties over number fields (see [1]). Even the special case $N=1$ and $d=4$ is enough to imply Merel's uniform boundedness of torsion points on elliptic curves [7. Though much work has been done on this problem for nearly 20 years, to date only non-uniform bounds are known.

As a first step, one might ask if Conjecture 1 holds in interesting families of dynamical systems. For example, in [13] Poonen conjectures a precise bound for quadratic polynomials over $\mathbb{Q}$, which can be viewed as a one-parameter family $f_{c}(z)=z^{2}+c:$

Conjecture 2. If $z_{0}, c \in \mathbb{Q}$ such that $z_{0}$ has primitive period $n$ for $f_{c}(z)=z^{2}+c$, then $n \leq 3$.

Received by the editors May 8, 2012 and, in revised form, September 14, 2012.

2010 Mathematics Subject Classification. Primary 37P05; Secondary 11R99.

The second and third authors' work was partially supported by NSF-DMS 1102858 . 
Even this special version of Conjecture 1 remains very much open. Morton [9] has shown that $n \neq 4$, Flynn, Poonen, Schaefer [2] showed that $n \neq 5$, and Stoll [16] proved that the Birch and Swinnerton-Dyer Conjecture implies $n \neq 6$. Given the difficulty of the question for quadratic polynomials, our Theorem 4.1 regarding a different one-parameter family of quadratic functions appears surprisingly strong.

Theorem. The rational map $\phi_{b}(z)=z+\frac{b}{z}$, where $b \in \mathbb{Q}$ has no rational points with primitive period $n \geq 5$.

It turns out that these two one-parameter families are different in a dynamically significant way. The quadratic functions $\phi_{b}$ considered above are all quadratic twists of the function $\phi(z)=z+1 / z$. This is not the case for the quadratic polynomials $f_{c}$ considered by Poonen and others.

In Theorem 2.9 we show that Conjecture 1 holds for families of twists of rational maps. More precisely, for any twist $\psi$ of a morphism of projective space $\phi$ defined over a number field $K$, the number of $K$-rational preperiodic points is uniformly bounded by a constant depending only on the map $\phi$, but independent of the twist. This statement and its proof are similar to a result for abelian varieties in 14 . There, Silverman shows that given an abelian variety $A$, for all but finitely many twists $A_{\xi}$ of $A$ the set of $K$-rational torsion points $A_{\xi}(K)_{\text {tors }}$ is contained in

$$
\left\{P \in A_{\xi}(\bar{K}) \text { : for some } f \in \text { Aut } A_{\xi}, f \neq \mathrm{id}, f(P)=P\right\} \text {. }
$$

We conclude with an outline of the paper and a survey of the techniques used.

Section 2; We provide background on Northcott's Theorem and twists of rational maps. We conclude with a proof of our main result, Theorem 2.9. which combines this background material with Galois-theoretic methods.

Section 3: We introduce the primary tool for understanding periodic points for morphisms of the projective line: dynatomic polynomials. We use arithmetic functions and elementary number theory to prove several lemmas about the coefficients of these polynomials for the family of maps $\phi_{k, b}(z)=k(z+b / z)$.

Section 4; Using the background in Section 3 and an application of the rational root theorem, we get the surprisingly strong Theorem 4.1 described above. Combining this theorem with results from [6], we give all possible graphs associated to rational preperiodic points for maps of the form $\phi_{b}(z)=z+b / z$; there are at most 6 rational preperiodic points for such maps.

Section 5: Using the same techniques, we get a similar theorem and graphical description of rational preperiodic point structures for the family of maps $\psi_{b}(z)=-(z+b / z)$.

\section{UNIFORM BOUNDS FOR FAMILIES OF TWISTS}

We begin with some background in arithmetic dynamics. Throughout, $K$ will be a number field, and we will state explicitly when results hold for more general fields.

Definition 2.1. We define

$$
\operatorname{Hom}_{d}^{N}(K)=\left\{\phi: \mathbb{P}^{N}(K) \rightarrow \mathbb{P}^{N}(K): \phi \text { is a morphism of degree } d^{N}\right\} .
$$


That is, $\phi$ is defined in each coordinate by homogeneous polynomials of degree $d$ with coefficients in $K$. (We follow the convention that $\operatorname{Hom}_{d}^{N}$ refers to $\operatorname{Hom}_{d}^{N}(\bar{K})$, and similarly for $\mathrm{PGL}_{N+1}$.)

The primary tool in our proof is a classic theorem of Northcott [11].

Theorem 2.2 (Northcott). Let $\phi \in \operatorname{Hom}_{d}^{N}(K)$. Then the set of preperiodic points $\operatorname{PrePer}\left(\phi, \mathbb{P}^{N}(\bar{K})\right)$ is a set of bounded height. In particular, $\operatorname{PrePer}\left(\phi, \mathbb{P}^{N}(K)\right)$ is a finite set, and more generally, for any $D>1$ the set

$$
\bigcup_{[L: K] \leq D} \operatorname{PrePer}\left(\phi, \mathbb{P}^{N}(L)\right)
$$

is finite.

Let $f \in \mathrm{PGL}_{N+1}$ act on the points of $\mathbb{P}^{N}(\bar{K})$ as a fractional linear transformation in the usual way. Then we define the morphism

$$
\phi^{f}=f \circ \phi \circ f^{-1} \text {. }
$$

Definition 2.3. Let $\phi, \psi \in \operatorname{Hom}_{d}^{N}(K)$. We say the morphisms are conjugate if there is some $f \in \mathrm{PGL}_{N+1}(\bar{K})$ such that $\phi^{f}=\psi$. They are conjugate over $K$ if there is some $f \in \mathrm{PGL}_{N+1}(K)$ such that $\phi^{f}=\psi$.

It is easily seen that $\left(\phi^{n}\right)^{f}=\left(\phi^{f}\right)^{n}$. Therefore if $P$ is periodic (resp. preperiodic) for $\phi$, then $f(P)$ is periodic (resp. preperiodic) for $\phi^{f}$ with the same period (resp. with the same period and same tail length). So conjugate maps have essentially the same dynamical behavior. However, if we are concerned with the arithmetic of the (pre)periodic points, we must be a bit more careful. For a map $\phi \in \operatorname{Hom}_{d}^{N}(K)$,

$$
\operatorname{Twist}(\phi / K)=\left\{\begin{array}{c}
K \text {-equivalence classes of maps } \psi \in \operatorname{Hom}_{d}^{N}(K) \\
\text { such that } \psi \text { is } \bar{K} \text {-equivalent to } \phi
\end{array}\right\} .
$$

An element $\psi \in \operatorname{Twist}(\phi / K)$ is called a twist of $\phi$.

Example 2.4. Let

$$
\phi(z / \sqrt{2} z)=z-\frac{2}{z} \text { and } \psi(z)=z-\frac{1}{z} .
$$

Also let $f(z)=z / \sqrt{2}$. One may check that $\phi^{f}(z)=\psi(z)$. So $\psi$ is a (quadratic) twist of $\phi$. Solving $\phi^{2}(z)=z$ gives the $\mathbb{Q}$-rational two-cycle $\{ \pm 1\}$. But $\psi$ does not have rational points of primitive period 2 ; solving $\psi^{2}(z)=z$ gives $\{ \pm 1 / \sqrt{2}\}$.

Definition 2.5. For any $\phi \in \operatorname{Hom}_{d}^{N}(\bar{K})$ define $\mathcal{A}_{\phi}$ to be the automorphism group of $\phi$, i.e.,

$$
\mathcal{A}_{\phi}=\left\{f \in \mathrm{PGL}_{N+1}(\bar{K}) \mid \phi^{f}=\phi\right\} .
$$

From [5, 12], $\mathcal{A}_{\phi}$ is well-defined as a finite subgroup of $\mathrm{PGL}_{N+1}(\bar{K})$.

We introduce some notation to make the statement and proof of Lemma 2.7 more succinct. Let $\phi \in \operatorname{Hom}_{d}^{N}(K)$ and $\psi \in \operatorname{Twist}(\phi / K)$, meaning there is an $f \in \mathrm{PGL}_{N+1}$ with $\phi^{f}=\psi$. Write $f=\left(a_{i j}\right)$, a matrix. At least one of the $a_{i j}$ is non-zero, say $a_{l m} \neq 0$. So we may also write $f=\left(a_{i j}^{\prime}\right)$ where $a_{i j}^{\prime}=\frac{a_{i j}}{a_{l m}}$, since this represents the same element of $\mathrm{PGL}_{N+1}$.

Definition 2.6. Let $K_{f}=K\left(a_{i j}^{\prime}\right)$ be the minimal field of definition for a given $f \in \mathrm{PGL}_{N+1}$ such that $\phi^{f}=\psi$, and let $L_{f}$ be the Galois closure of $K_{f}$. 
Lemma 2.7. For any $\psi \in \operatorname{Twist}(\phi / K)$ and any $f$ satisfying $\phi^{f}=\psi$,

$$
\left[K_{f}: K\right] \leq \# \mathcal{A}_{\phi}
$$

Proof. Choose $f \in \mathrm{PGL}_{N+1}$ such that $\phi^{f}=\psi$, and let $\sigma \in \operatorname{Gal}\left(L_{f} / K\right)$. Since $\psi$ is defined over $K$,

$$
\begin{aligned}
f \phi f^{-1} & =\left(f \phi f^{-1}\right)^{\sigma} \\
& =f^{\sigma} \phi^{\sigma}\left(f^{-1}\right)^{\sigma} \\
& =f^{\sigma} \phi\left(f^{-1}\right)^{\sigma}, \\
\phi & =f^{-1} f^{\sigma} \phi\left(f^{\sigma}\right)^{-1} f .
\end{aligned}
$$

Hence $f^{-1} f^{\sigma} \in \mathcal{A}_{\phi}$. Define the map

$$
\begin{aligned}
\rho: \operatorname{Gal}\left(L_{f} / K\right) & \rightarrow \mathcal{A}_{\phi}, \\
\sigma & \mapsto f^{-1} f^{\sigma} .
\end{aligned}
$$

Then

$$
\begin{aligned}
\rho(\sigma)=\rho(\tau) & \Leftrightarrow f^{-1} f^{\sigma}=f^{-1} f^{\tau} \\
& \Leftrightarrow f^{\sigma}=f^{\tau} \\
& \Leftrightarrow f=f^{\tau \sigma^{-1}} .
\end{aligned}
$$

So $\tau \sigma^{-1} \in \operatorname{Gal}\left(L_{f} / K_{f}\right)$ since it fixes $f$. Clearly if $\tau_{1} \sigma^{-1}=\tau_{2} \sigma^{-1}$ as elements of $\operatorname{Gal}\left(L_{f} / K_{f}\right)$, then they are equal as elements of $\operatorname{Gal}\left(L_{f} / K\right)$, and $\tau_{1}=\tau_{2}$. Hence $\rho$ is $\left[L_{f}: K_{f}\right]$-to- 1 , and we conclude that

$$
\left[L_{f}: K_{f}\right]\left[K_{f}: K\right]=\left[L_{f}: K\right] \leq\left[L_{f}: K_{f}\right]\left(\# \mathcal{A}_{\phi}\right)
$$

which gives the result since all of the extensions are finite.

Remark 2.8. If all elements of $\mathcal{A}_{\phi}$ are defined over $K$, then $\rho$ is in fact a group anti-homomorphism; that is, it is a homomorphism with the direction of group multiplication reversed. This is because $\sigma \tau \mapsto f^{-1} f^{\sigma \tau}$, but now $\left(f^{-1} f^{\tau}\right)^{\sigma}=f^{-1} f^{\tau}$. So we have

$$
\begin{aligned}
\rho(\sigma) \rho(\tau) & =f^{-1} f^{\sigma} f^{-1} f^{\tau}=f^{-1} f^{\sigma}\left(f^{-1} f^{\tau}\right)^{\sigma} \\
& =f^{-1} f^{\sigma}\left(f^{-1}\right)^{\sigma} f^{\tau \sigma}=f^{-1} f^{\tau \sigma} \\
& =\rho(\tau \sigma) .
\end{aligned}
$$

Inversion is also an anti-homomorphism, so the map $\rho^{-1}$, which maps each $\sigma \in$ $\operatorname{Gal}\left(L_{f} / K\right)$ to $\rho^{-1}(\sigma)$, is a homomorphism. The kernel of the anti-homomorphism is all $\sigma \in \operatorname{Gal}\left(L_{f} / K\right)$ such that $f^{\sigma}=f$. But this implies that $\operatorname{Gal}\left(L_{f} / K_{f}\right)$ is a normal subgroup of $\operatorname{Gal}\left(L_{f} / K\right)$, which implies that $K_{f} / K$ is Galois. Then $L_{f}=K_{f}$, and the map is injective. We conclude that $\operatorname{Gal}\left(L_{f} / K\right)$ is a subgroup of $\mathcal{A}_{\phi}$.

The following is now a straightforward consequence of Northcott's Theorem.

Theorem 2.9. Let $K$ be a number field and let $\phi \in \operatorname{Hom}_{d}^{N}(K)$. Then there is a uniform bound $B_{\phi}$ such that for all $\psi \in \operatorname{Twist}(\phi / K)$,

$$
\text { \# } \operatorname{PrePer}\left(\psi, \mathbb{P}^{N}(K)\right) \leq B_{\phi} \text {. }
$$


Proof. Given $\psi \in \operatorname{Twist}(\phi / K)$ and $f \in \mathrm{PGL}_{N+1}$ such that $\phi^{f}=\psi$, every $K$ rational preperiodic point for $\psi$ corresponds to a $K_{f}$-rational preperiodic point for $\phi$. From Lemma 2.7, $\left[K_{f}: K\right] \leq \# \mathcal{A}_{\phi}:=D$. By Northcott's Theorem,

$$
\bigcup_{[L: K] \leq D} \operatorname{PrePer}\left(\phi, \mathbb{P}^{N}\left(K_{f}\right)\right)
$$

is finite; call the cardinality of this set $B_{\phi}$. Then for every $\psi \in \operatorname{Twist}(\phi / K)$,

$$
\# \operatorname{PrePer}\left(\psi, \mathbb{P}^{N}(K)\right) \leq B_{\phi}
$$

Remark 2.10. In fact Lemma 2.7 holds over an arbitrary field $K$; hence Theorem 2.9 also holds when $K$ is a function field over a finite field.

Example 2.11. Suppose that $\phi(z): \mathbb{P}^{1}(K) \rightarrow \mathbb{P}^{1}(K)$ is a degree-2 morphism with a unique fixed point $P \in \mathbb{P}^{1}(\bar{K})$. Applying any Galois action to the equation $\phi(P)=P$ shows that any Galois conjugate of $P$ is also fixed by $\phi$. Hence, $P \in$ $\mathbb{P}^{1}(K)$. Choose a change of coordinates $f \in \mathrm{PGL}_{2}(K)$ moving $P$ to infinity. Then we may write

$$
\phi^{f}(z)=\frac{z^{2}+a z+b}{z+a} \text { for some } a, b \in K \text { with } b \neq 0 .
$$

The critical points of $\phi^{f}$ are $a \pm \sqrt{b}$, so conjugate by $g \in \mathrm{PGL}_{2}(K)$ which fixes infinity and moves the critical points to $\pm \sqrt{b}$, and we see that $\phi$ is conjugate over $K$ to a map of the form $\phi_{b}(z)=z+\frac{b}{z}$.

For any $b \in K^{*}, \phi_{b}$ is a quadratic twist of $\phi(z)=z+\frac{1}{z}$ with conjugating map $f_{b}=z \sqrt{b}$. So $f_{b} \in \mathrm{PGL}_{2}(L)$ with $[L: K] \leq 2$. Let $Q \in \mathbb{P}^{1}(K)$ be a $K$-rational preperiodic point for $\phi_{b}$. Then $f_{b}(Q)$ is an $L$-rational preperiodic point for $\phi$.

By Theorem 2.9. \# PrePer $\left(\phi_{b}, \mathbb{P}^{1}(K)\right) \leq B$ for some absolute bound $B$, independent of the parameter $b$. In Section 4, we show that for this twisted family of quadratic rational maps when $K=\mathbb{Q}$, the bound is actually 6 .

\section{Main tool: Dynatomic POlynomials}

For a morphism $\phi: \mathbb{P}^{1}(K) \rightarrow \mathbb{P}^{1}(K)$ (i.e. when the dimension $N=1$ ), we may choose $F, G \in K[z]$ such that $\phi(z)=F(z) / G(z)$ with $\operatorname{deg} \phi=\max \{\operatorname{deg} F, \operatorname{deg} G\}$. Hence we use the terms "morphism" and "rational map" interchangeably when the map is on the projective line.

In this section, we will write rational maps using homogeneous coordinates:

$$
\begin{aligned}
\phi: \mathbb{P}^{1} & \rightarrow \mathbb{P}^{1}, \\
{[X: Y] } & \mapsto[F(X, Y): G(X, Y)],
\end{aligned}
$$

where $F$ and $G$ are homogeneous polynomials of the same degree with no common factor. Then for $n>1$,

$$
\phi^{n}[X: Y]=\left[F_{n}(X, Y): G_{n}(X, Y)\right],
$$

where $F_{n}$ and $G_{n}$ are given recursively by

$$
F_{n}(X, Y)=F_{n-1}(F(X, Y), G(X, Y)) \text { and } G_{n}(X, Y)=G_{n-1}(F(X, Y), G(X, Y)) \text {. }
$$


Definition 3.1. The $n$-period polynomial of $\phi$ is

$$
\Phi_{\phi, n}(X, Y)=Y F_{n}(X, Y)-X G_{n}(X, Y) .
$$

The $n^{\text {th }}$ dynatomic polynomial of $\phi$ is the polynomial

$$
\Phi_{\phi, n}^{*}(X, Y)=\prod_{d \mid n} \Phi_{\phi, d}(X, Y)^{\mu\left(\frac{n}{d}\right)},
$$

where $\mu$ is the Mobius function. When the function $\phi$ is clear, we will suppress it from the notation, writing simply $\Phi_{n}^{*}$.

See [15, p. 151] for a proof that $\Phi_{n}^{*}(X, Y)$ is indeed a polynomial. Clearly it is then a homogeneous polynomial in $X$ and $Y$. Further, we let

$$
\nu_{2}(n)=\sum_{d \mid n} \mu\left(\frac{n}{d}\right) 2^{d}=\text { degree of } \Phi_{n}^{*} \text { for a quadratic rational map. }
$$

By construction, all points of period $d \mid n$ are roots of the $n$-period polynomial $\Phi_{n}$. One might hope that the roots of $\Phi_{n}^{*}$ are the points of primitive period $n$ (eliminating as roots points with period $d<n$ ). This isn't quite the case, but it is true that every point with primitive period $n$ is a root of $\Phi_{n}^{*}$, and that fact is enough for our purposes. See [15, Chapter 4] for details about dynatomic polynomials and their properties.

Lemma 3.2. The following products are positive powers of $k$ for $n>1$ :

(1) $\prod_{d \mid n}\left(k^{2^{d}-d-1}\right)^{\mu\left(\frac{n}{d}\right)}$.

(2) $\prod_{d \mid n}\left(k^{2^{d}-1}\right)^{\mu\left(\frac{n}{d}\right)}$.

(3) $\prod_{d \mid n}\left(k^{2^{d-1}}\right)^{\mu\left(\frac{n}{d}\right)}$.

(4) $\prod_{d \mid n}\left(k^{b(d)}\right)^{\mu\left(\frac{n}{d}\right)}$, where $b(d)=\left\lceil\frac{2\left(2^{d-1}-1\right)}{3}\right\rceil$.

Proof. The proofs will rely on facts about the properties of the arithmetic functions $\varphi$ and $\mu$, as well as the connection between them. We refer the interested reader to [8, Chapter 2] for these facts.

(1) Consider

$$
\begin{aligned}
\prod_{d \mid n}\left(k^{2^{d}-d-1}\right)^{\mu\left(\frac{n}{d}\right)} & =k^{\sum_{d \mid n} \mu\left(\frac{n}{d}\right)\left(2^{d}-d-1\right)} \\
& =k^{\sum_{d \mid n} \mu\left(\frac{n}{d}\right)\left(2^{d}-d\right)} .
\end{aligned}
$$

The last step follows from the fact that when $n>1$ the $\sum_{d \mid n} \mu\left(\frac{n}{d}\right)=0$. Recall that $\sum_{d \mid n} \mu\left(\frac{n}{d}\right) d=\varphi(n)$, where $\varphi(n)$ is the Euler totient function.

Also,

$$
\sum_{d \mid n} \mu\left(\frac{n}{d}\right) 2^{d} \geq 2^{n}-\sum_{d \mid n d \neq n} 2^{d} \geq 2^{n}-2^{n-1}=2^{n-1} .
$$


Hence

$$
\begin{aligned}
\sum_{d \mid n} \mu\left(\frac{n}{d}\right)\left(2^{d}-d\right) & =\sum_{d \mid n} \mu\left(\frac{n}{d}\right) 2^{d}-\sum_{d \mid n} \mu\left(\frac{n}{d}\right) d \\
& =\sum_{d \mid n} \mu\left(\frac{n}{d}\right) 2^{d}-\varphi(n) \\
& \geq \sum_{d \mid n} \mu\left(\frac{n}{d}\right) 2^{d}-n \\
& \geq 2^{n-1}-n .
\end{aligned}
$$

After taking the derivative of $2^{x-1}-x$, we see that the function is increasing as long as $x>1$. Hence $\sum_{d \mid n} \mu\left(\frac{n}{d}\right)\left(2^{d}-d\right)>0$.

(2) follows immediately from (1).

(3) follows immediately from equation (3.1), replacing $d$ by $d-1$ and using the fact that $n>1$.

(4) Now consider

$$
\sum_{d \mid n} \mu\left(\frac{n}{d}\right) b(d) \geq \sum_{d \mid n} \mu\left(\frac{n}{d}\right) \frac{2}{3}\left(2^{d-1}-1\right) .
$$

Multiplying the result from (3) by $\frac{2}{3}$ and using the fact that $\sum_{d \mid n} \mu(n / d)=0$ for $n>1$, we see that (4) is also positive.

Let $K$ be a number field and let

$$
\begin{aligned}
\phi_{k, b} & : \mathbb{P}^{1} \rightarrow \mathbb{P}^{1}, \\
{[X: Y] } & \mapsto\left[k\left(X^{2}+b Y^{2}\right): X Y\right]
\end{aligned}
$$

for some $b, k \in K^{*}$. A priori $\Phi_{n}^{*}$ is a polynomial in $X$ and $Y$. However, if we view $k$ and $b$ as parameters, we may ask if $\Phi_{n}^{*} \in \mathbb{Z}[k, b, X, Y]$. The following lemma will help us address this question.

Lemma 3.3. For every $n>1$,

(1) The coefficient of $X^{\nu_{2}(n)}$ in $\Phi_{\phi_{k, b} ; n}^{*}(X, Y)$ is a positive power of $k$ times $C_{n}(k)$, where $C_{n}(k)$ refers to the $n^{\text {th }}$ cyclotomic polynomial in the variable $k$.

(2) The coefficient of $Y^{\nu_{2}(n)}$ in $\Phi_{\phi_{k, b} ; n}^{*}(X, Y)$ is a positive power of $k$ times a positive power of $b$.

(3) Each monomial of $\Phi_{n}^{*}$ is divisible by $k$.

Proof. We have

$$
\begin{array}{ll}
F_{1}(X, Y)=k\left(X^{2}+b Y^{2}\right), & G_{1}(X, Y)=X Y, \\
F_{n}(X, Y)=k\left(F_{n-1}^{2}+b G_{n-1}^{2}\right), & G_{n}(X, Y)=F_{n-1} G_{n-1} .
\end{array}
$$

A simple induction argument shows that for $n>1$, we have $\operatorname{deg}_{X}\left(F_{n}\right)=\operatorname{deg}_{X}\left(G_{n}\right)+1$, and in fact $\operatorname{deg}_{X}\left(F_{n}\right)=2^{n}$ and $\operatorname{deg}_{X}\left(G_{n}\right)=2^{n}-1$.

The same arguments hold for $\operatorname{deg}_{Y} F_{n}$ and $\operatorname{deg}_{Y} G_{n}$. Now,

$$
\text { (coefficient of } \left.X^{2^{n}} \text { in } F_{n}\right)=k\left(\text { coefficient of } X^{2^{n-1}} \text { in } F_{n-1}\right)^{2} \text {, }
$$


so inductively this coefficient is $k^{2^{n}-1}$. Similarly, (coeff. of $X^{2^{n}-1}$ in $\left.G_{n}\right)=\left(\right.$ coeff. of $X^{2^{n-1}}$ in $\left.F_{n-1}\right)\left(\right.$ coeff. of $X^{2^{n-1}-1}$ in $G_{n-1}$ )

$$
=Y \prod_{i=0}^{n-1} k^{2^{i}-1}=Y k^{2^{n}-n-1} .
$$

Let $c_{d}$ be the coefficient of $X^{2^{d}}$ in $\Phi_{d}=Y F_{d}-X G_{d}$ and $c_{d}^{*}$ be the coefficient of $X^{\nu_{2}(d)}$ in $\Phi_{d}^{*}$ so that

$$
\begin{aligned}
c_{d} & =Y\left(k^{2^{d}-1}-k^{2^{d}-d-1}\right)=Y k^{2^{d}-d-1}\left(k^{d}-1\right), \\
c_{n}^{*} & =\prod_{d \mid n} c_{d}^{\mu\left(\frac{n}{d}\right)}=\prod_{d \mid n}\left(Y k^{2^{d}-d-1}\left(k^{d}-1\right)\right)^{\mu\left(\frac{n}{d}\right)} \\
& =\prod_{d \mid n} Y^{\mu\left(\frac{n}{d}\right)} \prod_{d \mid n}\left(k^{2^{d}-d-1}\right)^{\mu\left(\frac{n}{d}\right)} \prod_{d \mid n}\left(k^{d}-1\right)^{\mu\left(\frac{n}{d}\right)} .
\end{aligned}
$$

(Here, we use the definition of $\Phi_{n}^{*}$ and the fact that we know it is a polynomial in $X$ and $Y$.) When $n>1$, the first term is 1 , the second is a positive power of $k$ by Lemma 3.2 , and the third is $C_{n}(k)$, exactly as claimed.

Also,

$$
\text { (coefficient of } \begin{aligned}
\left.Y^{2^{n}+1} \text { in } \Phi_{n}\right) & =\left(\text { coefficient of } Y^{2^{n}} \text { in } F_{n}\right) \\
& =k\left(\text { coefficient of } Y^{2^{n-1}} \text { in } F_{n-1}\right)^{2},
\end{aligned}
$$

so inductively this coefficient is $k^{2^{n}-1} b^{2^{n-1}}$. So then

$$
\text { (coefficient of } \left.Y^{\nu_{2}(n)} \text { in } \Phi_{n}^{*}\right)=\prod_{d \mid n}\left(k^{2^{d}-1} b^{2^{d-1}}\right)^{\mu\left(\frac{n}{d}\right)},
$$

which is a positive power of $k$ times a positive power of $b$ by Lemma 3.2

The proof for the final claim is similar, but the algebraic details are messier. We sketch the main points here and leave the details for the reader. Inductively one may show that

$$
F_{n}(X, Y)=k^{a(n)} f_{n}(X, Y) \text { and } G_{n}(X, Y)=k^{b(n)} g_{n}(X, Y),
$$

where

$$
a(n)=\frac{2^{n}-(-1)^{n}}{3}, \quad b(n)=\left\lceil\frac{2\left(2^{n-1}-1\right)}{3}\right\rceil
$$

and $f_{n}, g_{n} \in \mathbb{Z}[k, b, X, Y]$. So then

$$
\Phi_{n}(X, Y)=k^{b(n)} \Psi_{n}(X, Y),
$$

where $\Psi_{n} \in \mathbb{Z}[k, b, X, Y]$. Then exactly as above, it follows that a positive power of $k$ divides each dynatomic polynomial $\Phi_{n}^{*}$.

Since $\Phi_{n}^{*}(X, Y)$ is homogeneous, we may dehomogenize in the usual way. We will write $\Phi_{n}^{*}(z)$ for the dehomogenized dynatomic polynomial. Note that the lead coefficient of $\Phi_{1}^{*}(z)=k-1=C_{1}(k)$ and the constant term is $b k$.

Mobius inversion gives $\prod_{d \mid n} \Phi_{\phi_{k, b}, d}^{*}(z)=\Phi_{\phi_{k, b}, n} \in \mathbb{Z}[k, b, z]$. In other words, $\Phi_{\phi_{k, b}, n}(z)$ factors over $\mathbb{Q}(k, b)$, so by Gauss's Lemma it factors over $\mathbb{Z}[k, b]$. Lemma 3.3 and the remark above show that the polynomials $\Phi_{\phi_{k, b}, d}^{*}(z)$ in the 
product each have content a non-negative power of $k$, meaning that $\Phi_{\phi_{k, b}, n}^{*}(z) \in$ $\mathbb{Z}[k, b, z]$.

Lemma 3.4. Let $n>1$. Then each monomial of the $n^{\text {th }}$ dynatomic polynomial $\Phi_{n}^{*}(X, Y)$ has the form $c_{i} X^{2 i} Y^{\nu_{2}(n)-2 i} b^{\frac{\nu_{2}(n)-2 i}{2}}$ where $c_{i} \in \mathbb{Z}[k]$.

Proof. We first show that for all $n \geq 1$,

$$
\begin{aligned}
F_{n}(X, Y) & =\sum_{i=0}^{2^{n-1}} c_{i} X^{2 i} Y^{2^{n}-2 i} b^{2^{n-1}-i} \quad \text { and } \\
G_{n}(X, Y) & =Y \sum_{j=1}^{2^{n-1}} d_{j} X^{2 j-1} Y^{2^{n}-2 j} b^{2^{n-1}-j}
\end{aligned}
$$

where $c_{i}, d_{j} \in \mathbb{Z}[k]$.

From equation (3.2), we see that $F_{1}$ and $G_{1}$ have the correct form. Assume $F_{n-1}$ and $G_{n-1}$ satisfy the claim above.

Consider

$$
F_{n}=k\left(\sum_{i=0}^{2^{n-2}} c_{i} X^{2 i} Y^{2^{n-1}-2 i} b^{2^{n-2}-i}\right)^{2}+k b\left(Y \sum_{j=1}^{2^{n-2}} d_{j} X^{2 j-1} Y^{2^{n-1}-2 j} b^{2^{n-2}-j}\right)^{2} .
$$

If we look at the first term monomial by monomial we get

$$
\begin{aligned}
\left(c_{i} X^{2 i} Y^{2^{n-1}-2 i} b^{2^{n-2}-i}\right) & \left(c_{j} X^{2 j} Y^{2^{n-1}-2 j} b^{2^{n-2}-j}\right) \\
& =c_{i} c_{j} X^{2(i+j)} Y^{2^{n}-2(i+j)} b^{2^{n-1}-(i+j)},
\end{aligned}
$$

so each monomial has the correct form. Now consider monomials from the second term:

$$
\begin{aligned}
k b Y^{2}\left(d_{i} X^{2 i-1} Y^{2^{n-1}-2 i} b^{2^{n-2}-i}\right) & \left(d_{j} X^{2 j-1} Y^{2^{n-1}-2 j} b^{2^{n-2}-j}\right) \\
& =k d_{i} d_{j} X^{2(i+j-1)} Y^{2^{n}-2(i+j-1)} b^{2^{n-1}-(i+j-1)},
\end{aligned}
$$

which has the correct form. This completes the proof for $F_{n}$, and the proof for $G_{n}$ is similar.

It follows immediately from equations $(3.3)$ and (3.4) that

$$
\Phi_{n}(X, Y)=Y F_{n}-X G_{n}=Y \sum_{i=0}^{2^{n}} e_{i} X^{2 i} Y^{2^{n}-2 i} b^{2^{n-1}-i} .
$$

We now compute the dynatomic polynomial:

$$
\begin{aligned}
\Phi_{n}^{*}(X, Y) & =\prod_{d \mid n}\left(Y \sum e_{i} X^{2 i} Y^{2^{d}-2 i} b^{2^{d-1}-i}\right)^{\mu\left(\frac{n}{d}\right)} \\
& =\prod_{d \mid n} Y^{\mu\left(\frac{n}{d}\right)} \prod_{d \mid n}\left(\sum e_{i} X^{2 i} Y^{2^{d}-2 i} b^{2^{d-1}-i}\right)^{\mu\left(\frac{n}{d}\right)} \\
& =\prod_{d \mid n}\left(\sum e_{i} X^{2 i} Y^{2^{d}-2 i} b^{2^{d-1}-i}\right)^{\mu\left(\frac{n}{d}\right)}
\end{aligned}
$$

where the last step follows because $n>1$. 
Let

$$
\alpha=\sum e_{i} X^{2 i} Y^{D_{\alpha}-2 i} b^{\frac{D_{\alpha}}{2}-i} \text { and } \beta=\sum f_{j} X^{2 j} Y^{D_{\beta}-2 j} b^{\frac{D_{\beta}}{2}-j} .
$$

Clearly the form is not affected if we add or subtract two such monomials. It is then easy to check that $\alpha \beta$ and $\frac{\alpha}{\beta}$ also have the correct form.

Lemma 3.5. For all $n \geq 1$, there exists a homogeneous polynomial $\psi_{n}(w, b) \in$ $\mathbb{Z}[w, b]$ such that $\psi_{n}\left(z^{2}, b\right)=\Phi_{n}^{*}(z, b)$.

Proof. From Lemma 3.4, when $n>1$ each monomial of $\Phi_{n}^{*}(z)$ has the form $c_{i} z^{2 i} b^{\frac{\nu_{2}(n)-2 i}{2}}$. A straightforward calculation shows that $\Phi_{1}^{*}(z)$ also has this form.

Now substitute $w=z^{2}$ to get $\Phi_{n}^{*}(w)$ with monomials of the form $c_{i} w^{i} b^{\frac{\nu_{2}(n)}{2}}-i$, which is homogeneous in $w$ and $b$, with degree $\frac{\nu_{2}(n)}{2}$.

Definition 3.6. Let $F(X, Y)$ be a homogeneous polynomial. We define $\ell(F)$ to be the leading coefficient of the dehomogenized polynomial $F(z, 1)$ and $c(F)$ to be the constant term of $F(z, 1)$.

Lemma 3.7. Let

$$
\begin{aligned}
\phi_{b}(X, Y): \mathbb{P}^{1} & \rightarrow \mathbb{P}^{1} \\
(X, Y) & \mapsto\left(X^{2}+b Y^{2}, X Y\right) .
\end{aligned}
$$

Then

$$
\ell\left(\Phi_{n}^{*}(X, Y)\right)= \begin{cases}p & \text { if } n=p^{e}, e \geq 1, \\ b & \text { if } n=1, \\ 1 & \text { otherwise }\end{cases}
$$

and $c\left(\Phi_{n}^{*}\right)$ is a non-negative power of $b$.

Proof. The result for $n=1$ follows from the remark after Lemma 3.3 .

For $n>1$, we see from Lemma 3.3 part (1) that $\ell\left(\Phi_{n}^{*}\right)$ is a non-negative power of $k$ times $C_{n}(k)$, where $C_{n}(k)$ is the $n^{\text {th }}$ cyclotomic polynomial in the variable $k$. The result follows from evaluating this at $k=1$. Similarly, the result for $c\left(\Phi_{n}^{*}\right)$ follows from part (2) of Lemma 3.3 and evaluating at $k=1$. (See, for example, [4, p. 280 points 2 . and 4.] to evaluate the cyclotomic polynomials at $k=1$.)

\section{The EXPLICIT BOUND}

In this section, we find an explicit uniform bound for the number of $\mathbb{Q}$ preperiodic points for a one-parameter family of quadratic rational maps; namely, the quadratic maps with a unique fixed point. The existence of such a bound follows immediately from Theorem [2.9, so the content of this work is in finding the bound explicitly. By construction, a rational point $\left(z_{0}, b_{0}\right)$ on the variety $V\left(\Phi_{n}^{*}(z, b)\right)$ corresponds to a quadratic rational map $\phi(z)=z+\frac{b_{0}}{z}$ and a rational point $z_{0}$ of period $n$ for $\phi$. Note that $b_{0} \neq 0$, since that value does not give a degree 2 rational map.

By Lemma 3.5. we may substitute $w=z^{2}$ in $\Phi_{n}^{*}$, and the resulting polynomial $\psi_{n}(w, b) \in \mathbb{Z}[w, b]$ is homogeneous in $w$ and $b$. So if $V\left(\Phi_{n}^{*}(z, b)\right)$ has a rational point $\left(z_{0}, b_{0}\right)$ with $b_{0} \neq 0$, then $V\left(\psi_{n}(w, b)\right)$ has a rational point $\left(z_{0}^{2}, b_{0}\right)$. Since $\psi_{n}(w, b)$ is homogeneous, we may equivalently ask if $\psi_{n}(w, 1)$ has a rational root. 
Theorem 4.1. The rational map $\phi(z)=z+\frac{b}{z}$, where $b \in \mathbb{Q}$ has no rational points with primitive period $n \geq 5$.

Proof. From Lemma 3.7, we know that for $n>1$ the lead coefficient of $\psi_{n}(w, 1) \in$ $\mathbb{Z}[w]$ is either 1 (if $n$ is not a prime power) or $p$ (if $n=p^{e}$ ) and the constant term is 1 . The only rational roots of such a polynomial are \pm 1 when $n$ is not a prime power, and \pm 1 or $\pm \frac{1}{p}$ when $n$ is a power of a prime.

In either case, we can have no more than four rational roots of $\psi_{n}$, which means no more than four rational points are on any given cycle, and this is independent of the parameter $b$.

We now combine Theorem 4.1 with earlier work on the two-parameter family $\phi(z)=k z+\frac{b}{z}$ to find exactly what primitive periods are possible for rational periodic points.

Corollary 4.2. If $P \in \mathbb{P}^{1}(\mathbb{Q})$ is a periodic point for $\phi(z)=z+\frac{b}{z}$ where $b \in \mathbb{Q}$, then $P$ is either the point at infinity or a point of primitive period 2 .

Proof. From Theorem 4.1, we know that the primitive period of $P$ must be less than 5.

From [6, Theorem 4], a map of the form $\phi(z)=k z+\frac{b}{z}$ with $k, b \in \mathbb{Q}^{*}$ has a rational point of primitive period 4 if there is some $m \in \mathbb{Q} \backslash\{0, \pm 1\}$ such that $k=2 m /\left(m^{2}-1\right)$ and $b=-m /\left(m^{4}-1\right)$. In the proof of that theorem, we see that the map has a rational point of primitive period 4 if and only if we can find a rational point on a certain algebraic curve. The parameterization of the curve given in the proof shows that in fact the condition $k=2 m /\left(m^{2}-1\right)$ is necessary. However, there is no such $m$ with $1=2 m /\left(m^{2}-1\right)$. We conclude that $P$ has primitive period less than 4 .

Similarly, 6, Theorem 3] says that if $\phi(z)=k z+\frac{b}{z}$ with $k, b \in \mathbb{Q}^{*}$, then $\phi(z)$ has no rational point of primitive period 3. Hence, $P$ has primitive period one or two.

By construction, $P$ is a fixed point if and only if $P$ is the point at infinity. The only other possibility is a rational point of primitive period 2 .

To finish finding the exact bound on the number of rational preperiodic points for a map of the form $\phi(z)=z+\frac{b}{z}$, we introduce a bit more notation.

Definition 4.3. Let $m$ and $n$ be positive integers. Given a rational map $\phi$ and a point $P$ that is strictly preperiodic for $\phi$ (in other words, $P$ is preperiodic but not periodic), we say that $P$ has type $m_{n}$ if $P$ enters a cycle of primitive period $m$ after $n$ iterations. That is, $\phi^{n+m}(P)=\phi^{n}(P)$, where $m \geq 1$ and $n \geq 1$ are the smallest such integers.

Corollary 4.4. Let $\phi(z)=z+\frac{b}{z} \in \mathbb{Q}(z)$ with $b \neq 0$. Then $\phi$ has either 2 , 4 , or 6 rational preperiodic points.

Proof. First note that for every non-zero $b \in \mathbb{Q}$, the point at infinity is fixed, and $\phi(0)$ is infinity. Hence, every $\phi_{b}$ has a rational fixed point and a rational point of type $1_{1}$.

Applying [6, Proposition 6], we see that $\phi$ has a rational point of type $1_{2}$ if and only if $b=-c^{2}$ for some $c \in \mathbb{Q}^{*}$. However, all of these maps are conjugate over $\mathbb{Q}$, so take $b=-1$ as a representative. From [6, Propositions 7 and 8], we conclude that $\phi$ has no rational points of type $1_{n}$ for $n>2$. 
Applying [6, Proposition 2], we see that $\phi$ has a rational point of primitive period 2 if and only if $b=-2 c^{2}$ for some $c \in \mathbb{Q}^{*}$. Again, all such maps are conjugate over $\mathbb{Q}$, so we take $b=-2$ as a representative. It is a simple matter to check that in this case, $\phi_{b}$ has two rational points of type $2_{1}$. (See Figure 1.) From [6, Proposition 8], we conclude that $\phi_{b}$ has no rational points of type $2_{n}$ for $n>1$.

Finally, [6, Proposition 9] says that $\phi_{b}$ has both rational points of type $1_{2}$ and rational points of primitive period 2 if and only if we can solve $1=1 /\left(x^{2}-1\right)$ with $x \in \mathbb{Q} \backslash\{0, \pm 1\}$. Evidently, this is not possible. Hence for $b=-1$ (and all $\mathbb{Q}$-conjugate maps), $\phi_{b}$ has four rational preperiodic points. Similarly, for $b=-2$ (and all $\mathbb{Q}$-conjugate maps), $\phi_{b}$ has six rational preperiodic points. There are no $b$ values for which $\phi_{b}$ has more than six rational preperiodic points.

We provide a graphical representation of all possible structures for rational preperiodic points for the family $\phi(z)=z+\frac{b}{z}$. In these graphs, the vertices represent points in $\mathbb{P}^{1}(\mathbb{Q})$ and an arrow from vertex $P$ to vertex $Q$ indicates $\phi(P)=Q$.

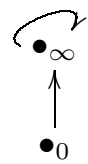

(a) $b=1$

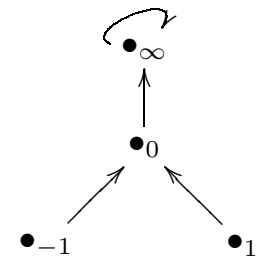

(b) $b=-1$

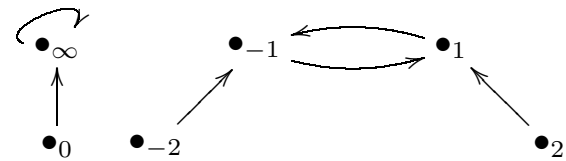

(c) $b=-2$

Figure 1. All possible rational preperiodic graphs for $\phi_{b}(z)=z+\frac{b}{z}$.

\section{Another FAMily of twists}

We conclude with an abbreviated analysis of the possible preperiodic structures for another family of twists. Lemma 3.3 says the lead coefficient of the dynatomic polynomials are powers of $k$ times a cyclotomic polynomial in $k$. With the help of [3, Proposition 1], we can evaluate cyclotomic polynomials at roots of unity. Hence, we consider $k=-1$. The proofs are similar to those in Section 4, so the details will be sketched here.

We now consider the maps $\psi_{b}(z)=-\left(z+\frac{b}{z}\right)$. Again, each $\psi_{b}$ is conjugate to $\psi_{1}$ via the map $f(z)=z \sqrt{b}$. The family of twists is distinct from the one already considered, since $\psi_{b}$ has two distinct finite fixed points at $\pm \sqrt{-b / 2}$ and a third fixed point at infinity.

Note that Lemmas 3.3. 3.4 and 3.5 apply to the family $\psi_{b}$, as we are taking $k=-1$.

Lemma 5.1. Let

$$
\begin{aligned}
\psi_{b}(X, Y): \mathbb{P}^{1} & \rightarrow \mathbb{P}^{1}, \\
(X, Y) & \mapsto\left(-\left(X^{2}+b Y^{2}\right), X Y\right) .
\end{aligned}
$$


Then

$$
\ell\left(\Phi_{n}^{*}(X, Y)\right)= \begin{cases} \pm p & \text { if } n=2 p^{e}, e \geq 1, p \text { prime } \\ -2 & \text { if } n=1, \\ \pm 1 & \text { otherwise }\end{cases}
$$

and $c\left(\Phi_{n}^{*}\right)$ is a non-negative power of $b$.

Proof. The case $n=1$ is found by computation. By Lemma 3.3, the lead coefficient of $\psi_{b}$ is some power of $k$ times a cyclotomic polynomial. We apply [3. Proposition 1] to evaluate the cyclotomic polynomials at $k=-1$, yielding the result.

Proposition 5.2. Let $\psi_{b}(z)=-\left(z+\frac{b}{z}\right) \in \mathbb{Q}(z)$ with $b \neq 0$. Then $\psi_{b}$ has either 2 or 4 rational preperiodic points.

Proof. A proof identical to Theorem 4.1 using Lemma 5.1 shows there can be no rational points of primitive period $n \geq 5$, and we apply [6] Theorems 3 and 4] to see that there are no rational points of primitive period 3 or 4 . From [6, Proposition 2], we conclude that no value of $b \in \mathbb{Q}^{*}$ gives a rational map with a rational point of primitive period 2 . Hence, we need only consider fixed points and points of type $1_{n}$ for $n \geq 1$.

For every $b \in \mathbb{Q}^{*}$, there is a rational fixed point at $\infty$ and a rational point of type $1_{1}$ at 0 . By [6. Proposition 1], $\psi_{b}$ has rational finite fixed points if and only if $b=-2 c^{2}$. Since all such maps are conjugate over $\mathbb{Q}$, we take $b=-2$ as a representative. There are no other type $1_{1}$ rational points. Applying [6, Proposition 6 ] we have rational points of type $1_{2}$ if and only if $b=-c^{2}$. Again, all such maps are conjugate over $\mathbb{Q}$, so we take $b=-1$ as a representative. By [6, Proposition 7 and 8$]$ there are no rational points of type $1_{n}$ for $n>2$. See Figure 2

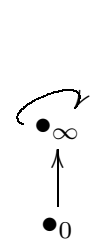

(a) $b=2$

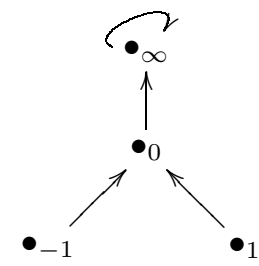

(b) $b=-1$

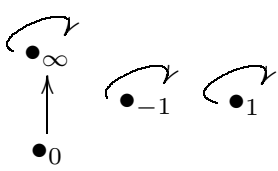

(c) $b=-2$

Figure 2. All possible rational preperiodic graphs for $\psi_{b}(z)=-\left(z+\frac{b}{z}\right)$.

\section{ACKNOWLEDGMENTS}

The authors would like to thank the Institute for Computational and Experimental Research in Mathematics for a productive and enjoyable semester during which much of this project was completed. The authors benefited greatly from productive conversations with workshop participants, including Holly Krieger, William Gignac, Frank Paladino, Chi-hao Wang, Andrew Bridy, Kevin Doerksen, Jacqueline Anderson, Jan-Li Lin, Katherine Stange and especially Mike Zieve, who suggested the full strength of Theorem 2.9. Thanks also to Joseph Silverman, Xander Faber, Bianca Viray, and the referee for numerous helpful comments. 


\section{REFERENCES}

[1] Najmuddin Fakhruddin, Questions on self maps of algebraic varieties, J. Ramanujan Math. Soc. 18 (2003), no. 2, 109-122. MR1995861 (2004f:14038)

[2] E. V. Flynn, Bjorn Poonen, and Edward F. Schaefer, Cycles of quadratic polynomials and rational points on a genus-2 curve, Duke Math. J. 90 (1997), no. 3, 435-463, DOI 10.1215/S0012-7094-97-09011-6. MR 1480542 (98j:11048)

[3] R. P. Kurshan and A. M. Odlyzko, Values of cyclotomic polynomials at roots of unity, Math. Scand. 49 (1981), no. 1, 15-35. MR645087(83k:12006)

[4] Serge Lang, Algebra, 3rd ed., Graduate Texts in Mathematics, vol. 211, Springer-Verlag, New York, 2002. MR1878556(2003e:00003)

[5] Alon Levy, The space of morphisms on projective space, Acta Arith. 146 (2011), no. 1, 13-31, DOI 10.4064/aa146-1-2. MR2741188 (2012d:37211)

[6] Michelle Manes, $\mathbb{Q}$-rational cycles for degree-2 rational maps having an automorphism, Proc. Lond. Math. Soc. (3) 96 (2008), no. 3, 669-696, DOI 10.1112/plms/pdm044. MR2407816 (2009a:14029)

[7] Loïc Merel, Bornes pour la torsion des courbes elliptiques sur les corps de nombres (French), Invent. Math. 124 (1996), no. 1-3, 437-449, DOI 10.1007/s002220050059. MR1369424 (96i:11057)

[8] Steven J. Miller and Ramin Takloo-Bighash, An invitation to modern number theory, with a foreword by Peter Sarnak, Princeton University Press, Princeton, NJ, 2006. MR2208019 (2006k:11002)

[9] Patrick Morton, Arithmetic properties of periodic points of quadratic maps. II, Acta Arith. 87 (1998), no. 2, 89-102. MR1665198 (2000c:11103)

[10] Patrick Morton and Joseph H. Silverman, Rational periodic points of rational functions, Internat. Math. Res. Notices 2 (1994), 97-110, DOI 10.1155/S1073792894000127. MR1264933 (95b:11066)

[11] D. G. Northcott, Periodic points on an algebraic variety, Ann. of Math. (2) 51 (1950), 167177. MR0034607 (11,615c)

[12] Clayton Petsche, Lucien Szpiro, and Michael Tepper, Isotriviality is equivalent to potential good reduction for endomorphisms of $\mathbb{P}^{N}$ over function fields, J. Algebra 322 (2009), no. 9, 3345-3365, DOI 10.1016/j.jalgebra.2008.11.027. MR2567424(2011e:14023)

[13] Bjorn Poonen, The classification of rational preperiodic points of quadratic polynomials over Q: a refined conjecture, Math. Z. 228 (1998), no. 1, 11-29, DOI 10.1007/PL00004405. MR 1617987 (99j:11076)

[14] Joseph H. Silverman, Lower bounds for height functions, Duke Math. J. 51 (1984), no. 2, 395-403, DOI 10.1215/S0012-7094-84-05118-4. MR.747871 (87d:11039)

[15] Joseph H. Silverman, The arithmetic of dynamical systems, Graduate Texts in Mathematics, vol. 241, Springer, New York, 2007. MR2316407 (2008c:11002)

[16] Michael Stoll, Rational 6-cycles under iteration of quadratic polynomials, LMS J. Comput. Math. 11 (2008), 367-380, DOI 10.1112/S1461157000000644. MR2465796 (2010b:11067)

Department of Mathematics, University of British Columbia, Vancouver, BC, Canada V6T124

E-mail address: levy@math.ubc.ca

Department of Mathematics, University of Hawain at Manoa, Honolulu, Hawail 96822

E-mail address: mmanes@math.hawaii.edu

Department of Mathematics, University of Hawail at Manoa, Honolulu, Hawail 96822 E-mail address: bat7@hawaii.edu 